\title{
Nonlinear Companding Scheme to Reduce PAPR
}

\author{
P. T. Sivagurunathan*, P. Nithya, N. Mahendran and V. Kavitha \\ Department of Electronics and communication engineering, M. Kumarasamy College of Engineering, \\ Karur, Thalavapalayam - 639113, Tamil Nadu, India; sivagurunathanpt.ece@mkce.ac.in, \\ nithyap.ece@mkce.ac.in, mahendrann.ece@mkce.ac.in, kavithav.ece@mkce.ac.in
}

\begin{abstract}
Objectives: To reduce the PAPR in OFDM signal. Methods/Statistical Analysis: The foremost problem found in OFDM is the high PAPR (Peak to Average Power Ratio). The variety of processes worned to diminish the high PAPR is: Partial Transmit Sequence (PTS) Coding, Clipping, m-Law, Selective Mapping (SLM), Exponential Companding (EC) etc. Findings: However, these PAPR reduction techniques have some disadvantages, such as Higher Modulation Order, Degradation of the BER and SNR performance, increasing of Average Power etc. A new Nonlinear Companding technique is proposed which aims at compressing the large amplitude signals and at the same time sustaining the normal power as stable. The Simulation results show that the proposed Companding method possibly provides an improved scheme recital in stipulations of PAPR lessening, and BER recital. Application/Improvements: Amplifier will not get damage.
\end{abstract}

Keywords: Exponential Companding (EC), PAPR (Peak to Average Power Ratio), Partial Transmit Sequence (PTS), Selective Mapping (SLM)

\section{Introduction}

OFDM has gained popularity owing to the reward of high spectral effectiveness, forcefulness to frequency discriminating (selecting) departure canal (channel) and effortless execution with Fast Fourier Transform ${ }^{1,2}$ in a number of relevance jointly with standard IEEE 802.16d for Digital Audio Broadcasting, Wireless Metropolitan Area Networks and the standard IEEE 802.11a for Terrestrial Digital Video Broadcasting and Wireless Local Area Networks.

Nevertheless, a few difficulties are still unreciprocated in the existing arrangements (systems). This large PAPR strengthens the send out control (power) amplifier to contain a huge back-off so that we can make sure that the linear amplification of the gestures, which drastically diminishes the effectiveness of the amp. There are several clarifications are included in the existing system to avoid this large PAPR, and it can be separated in two types: First type is used to diminish the chance of produc- ing large PAPR gestures prior to proceeding the multicarrier inflection (modulation), for instance Block coding ${ }^{3}$, Selective Mapping, and Partial Transmit Sequences. The another type is used to contract with the gestures behind the multicarrier inflection, like extracting (clipping) and the companding change (transform), amongst the easiest and most commonly used for dropping the PAPR is clipping. However, it causes extra clipping blare (noise). Also, this extracting (clipping) blare turn out to be very important by means of high modulation information's and badly disgrace the system recital, which creates the companding further appropriate for high information rates appliances. In, Wang initially projected the $\mu$-law companding method depends on communication (speech) processing, moreover this method illustrates enhanced narration compared with the clipping method. The Peak Average Power Ratio is condensed at the cost of an increased usual power by means of this companding law. Later on, a nonlinear companding method that is exponential companding were introduced to rise above the difficulty of rising 
usual (average) power and to comprise good PAPR lessening. This method converts the Rayleigh dispersed OFDM gesture to a equally (uniformally) dispersed gesture and it attains improved system presentation when compared to $\mu$-law companding method. Nevertheless, the division of huge amplitude gestures is improved by means of equal companding method. Therefore, by means of power amplifier with great nonlinearity we can able to expect that BER presentation is reduced at that time of OFDM.

In this article, the proposed companding method largely is focusing on reducing the large gestures, while keeping the normal power stable by appropriately selecting the transform factors. At last, the simulation consequence confirms to the planned method proposes a improved PAPR lessening and BER presentation.

The remaining sections of this paper are arranged as; Section 2 demonstrates an OFDM method characteristics and difficulty of large PAPR. Section 3 establishes the planned method which provides an excellent BER presentation. In Section 4, the recital of the planned method is matched up to with the various modulation methods during simulation. At last, conclusions are specified in Section 5 .

\section{OFDM Scheme with Problem Definition}

Figure 1 illustrates the common building block of OFDM system with the companding method, the entire bandwidth of this system is separated into various sub-channels with fine bandwidth, and information cryptogram (symbols) usually varied by means of Phase Shift Keying Or QAM which are send out separately on the subcarriers. Allow $\mathrm{N}$ to indicate the quantity of sub-carriers worned for similar (parallel) data broadcasting and $\mathrm{X}_{\mathrm{k}}(0 \leq \mathrm{k} \leq \mathrm{N}-1)$ indicates the kth complex adjusted (modulated) character within a piece of $\mathrm{N}$ information. The give up of $\mathrm{x}_{\mathrm{n}}$-point IFFT of $\mathrm{X}_{\mathrm{k}}$ are given by:

$$
x_{n}=\frac{1}{\sqrt{N}} \sum_{k=0}^{N-1} X_{k} \exp \left(\frac{j 2 \prod k n}{N}\right)
$$

Subsequently, the signal power of OFDM for $x_{n}$ is specified as,

$$
|x n|^{2}=\frac{1}{N} \sum_{m=0}^{N-1} \sum_{k=0}^{N-1} X_{m} X_{k} \exp \left(\frac{j .2 \prod(m-k)}{N}\right)
$$

and the PAPR by means of power is illustrates as,

$$
P A P R=10 \log _{10} \frac{\operatorname{Max}\left\{\left|x_{n}\right|^{2}\right\}}{E\left\{\left|x_{n}\right|^{2}\right\}}(d B)
$$

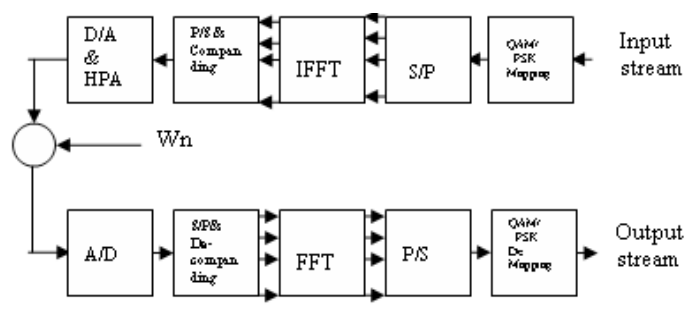

Figure 1. Generic illustrations of an OFDM method with the proposed transform.

\section{Proposed Scheme}

Here in this part, we offer a work of fiction as non-linear companding method to get better BER presentation effectively and to lessen the PAPR OFDM gestures. Furthermore, the latest method provides benefits of keeping a stable average power during the companding process. As a result, the effectiveness of an amplifier can be able to improve. Suppose if the input data ciphers are statistically self-determining and equally dispersed. With the help of central limit hypothesis, $\mathrm{x}_{\mathrm{n}}$ can be represented as a complex Gaussian method if the amount of sub-carriers is $\mathrm{N}$ huge (e.g.N $\geq 64$ ). Now, consider that the $\mathrm{x}_{\mathrm{n}}$ has a " 0 " mean and variance $\left(\sigma^{2}\right)$, therefore the magnitude $\left|x_{n}\right|$ contains a Rayleigh sharing by means of Probability Distribution Function which is represented by,

$$
\left.f\right|_{x n} \mid(x)=\frac{2 x}{\sigma^{2}} \exp \left(-\frac{x^{2}}{\sigma^{2}}\right), \mathrm{x} \geq 0
$$

The CDF of $\left|x_{n}\right|$ can be characterized as,

$$
\begin{aligned}
& \left.F\right|_{x n} \mid=\operatorname{Pr} o p\left\{\left|x_{n}\right| \leq x\right\} \\
& =1-\exp \left(-\frac{x^{2}}{\sigma^{2}}\right), \mathrm{x} \geq 0
\end{aligned}
$$

The planned companding method is specified by

$$
t_{n}=h\left(x_{n}\right)
$$


By considering the phase of input signal, the $\mathrm{h}(\mathrm{x})$ is detailed as,

$$
\begin{gathered}
h(x)=\operatorname{sgn}(x) F_{|t t|}^{-1} F_{|x| l \mid}(x) \\
h(x)=\left\{\begin{array}{l}
x \\
\operatorname{sgn}(x) \cdot \sqrt{6 \sigma}\left(\frac{2}{3}-\frac{1}{2} \exp \left(\frac{1}{6}-\frac{|x|^{2}}{\sigma^{2}}\right)\right) \quad|x|>\frac{\sigma}{\sqrt{6}}
\end{array}\right.
\end{gathered}
$$

De-companding task at the receiver is given by,

$$
h^{-1}(x)= \begin{cases}x \quad|x| \leq \frac{\sigma}{6} & |x|>\frac{\sigma}{\sqrt{6}} \\ \operatorname{sgn}(x) \cdot \frac{\sigma}{6} \sqrt{6-36 \cdot \ln \left(\frac{4 \sigma-\sqrt{6}|x|}{3 \sigma}\right)} & \end{cases}
$$

As a result of Equation (3), the average power of the planned method can be exposed as:

$$
\begin{aligned}
& P A P R=10 \log _{10} \frac{\operatorname{Max}\left\{\left|x_{n}\right|^{2}\right\}}{E\left\{\left|x_{n}\right|^{2}\right\}}(d B) \\
& =10 \log _{10} \frac{A^{2}}{\sigma^{2}}=6.00(\mathrm{~dB})
\end{aligned}
$$

Figure 2 and 3 represents the outline of Exponential Companding (EC) and Nonlinear companding methods. From this outline, it can be attained with the aim of the Nonlinear method can provide improved PAPR lessening in contrast to the EC method.

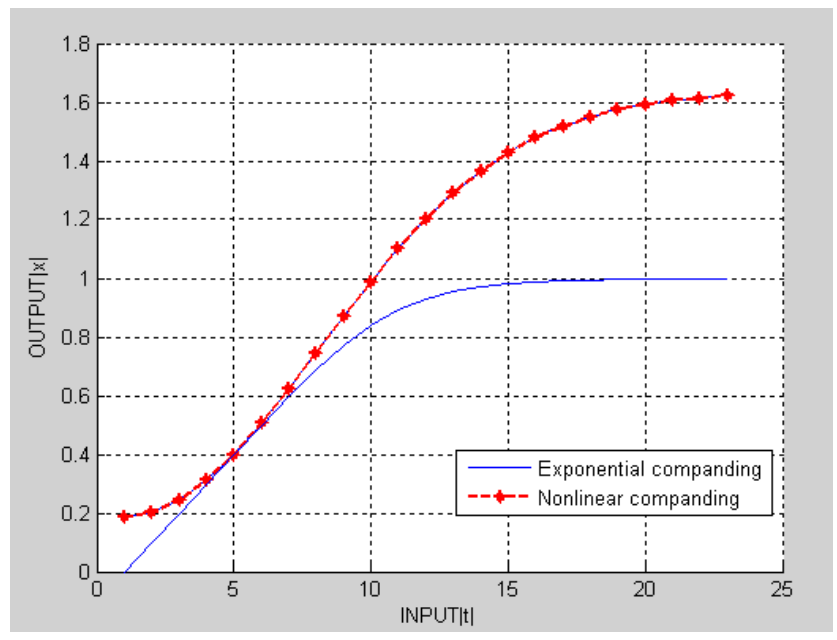

Figure 2. Profiles of different companding schemes.

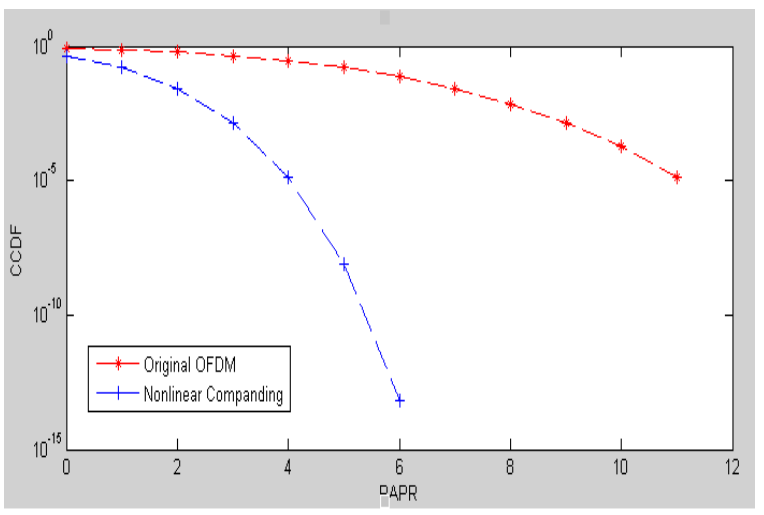

Figure 3. Original OFDM signal and companded signals.

\section{Performance Estimation}

The fading channels and AWGN are considered in the HPA by simulations, in order to estimate the lessening of Power average and presentation of BER of the designed system with the conventional system (Exponential Companding). Moreover, the majority of radio schemes frequently utilize large (High) Power in the transmitting side in order to get enough broadcast power. That's why, a single WLAN model number AP 1093 formed by RF incorporated Corp. is engaged at this time as a result, the enough broadcast power be able to be accomplished. Moreover, we assumed that $\mathrm{N}=64$ of the OFDM system make use of the aspect of oversampling $\mathrm{L}=4$ and an accidentally created input data.

As per Figure 4-7 we can state that a better BER is achieved in the designed method. From Table 1 and 2, the PAPR for designed method is $6 \mathrm{~dB}$ and $11 \mathrm{~dB}$ for conventional method.

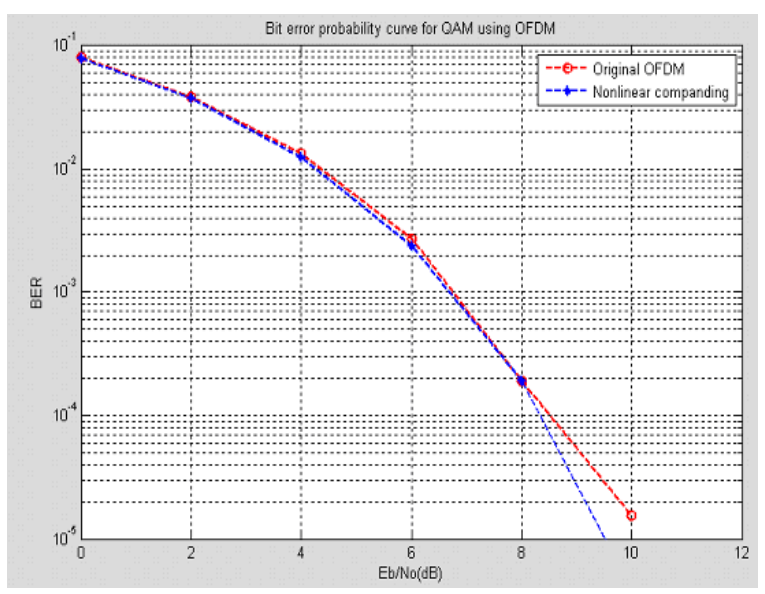

Figure 4. OFDM BER narration with proposed system (nonlinear companding )-AWGN channel using QAM. 


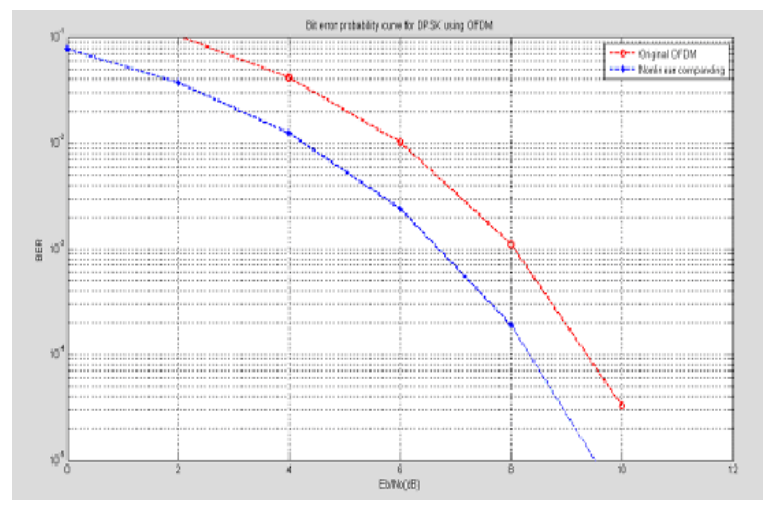

Figure 5. OFDM BER narration with proposed system (nonlinear companding )-AWGN channel using

DPS.

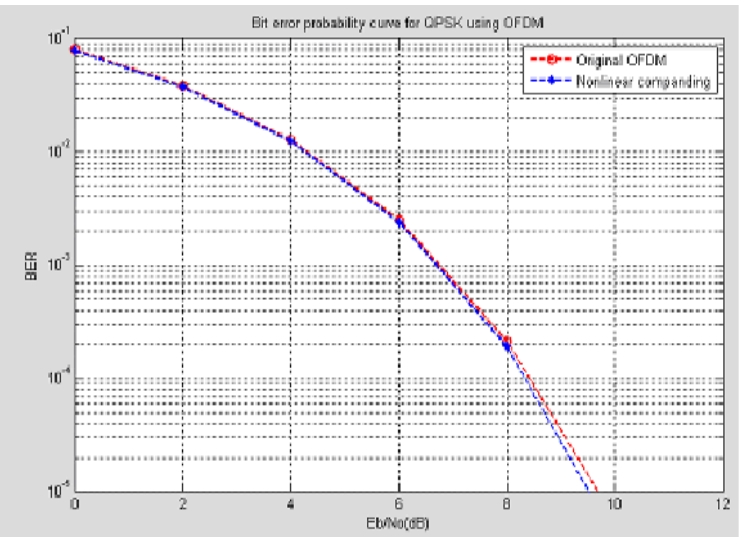

Figure 6. OFDM BER narration with proposed system (nonlinear companding )-AWGN channel using BPS.

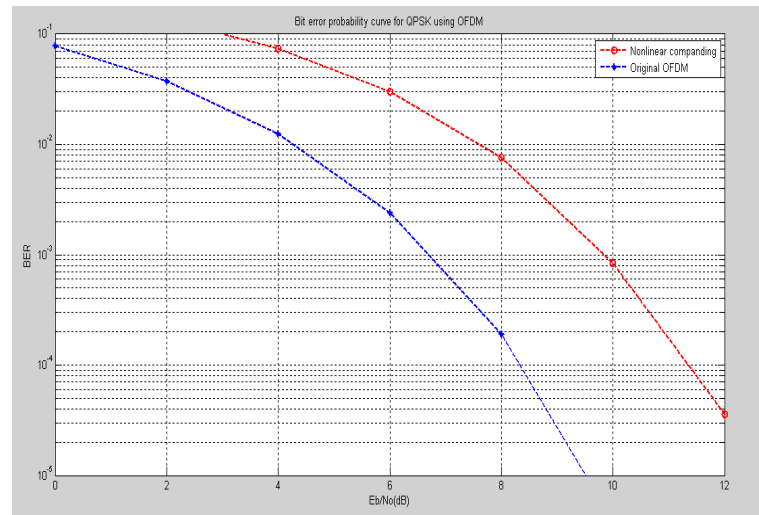

Figure 7. OFDM BER narration with proposed system (nonlinear companding )-AWGN channel using QPS.

Figure 8 depicts the original OFDM BER narration with proposed system (nonlinear companding)- Rayleigh channel using BPSK. As a result, we can declare that the presentation of the BER of planned method is moreover
Table 1. Comparison of BER with various modulation schemes

\begin{tabular}{|c|c|c|c|c|c|c|}
\hline \multirow{3}{*}{ Schemes } & \multicolumn{6}{|c|}{$\mathrm{Eb} / \mathrm{NO}$} \\
\hline & \multicolumn{2}{|l|}{ QAM } & \multicolumn{2}{|l|}{ BPSK } & \multicolumn{2}{|l|}{ DPSK } \\
\hline & $\begin{array}{l}\mathrm{BER}= \\
10-3\end{array}$ & $\begin{array}{l}\mathrm{BER}= \\
10-4\end{array}$ & $\begin{array}{l}\mathrm{BER}= \\
10-3\end{array}$ & $\begin{array}{l}\mathrm{BER}= \\
10-4\end{array}$ & $\begin{array}{l}\mathrm{BER}= \\
10-3\end{array}$ & $\begin{array}{l}\mathrm{BER}= \\
10-4\end{array}$ \\
\hline $\begin{array}{l}\text { Original } \\
\text { OFDM }\end{array}$ & 6.97 & 8.50 & 6.80 & 8.20 & 6.99 & 8.20 \\
\hline $\begin{array}{l}\text { Nonlinear } \\
\text { companding }\end{array}$ & 6.99 & 8.60 & 6.82 & 8.40 & 8.00 & 9.10 \\
\hline
\end{tabular}

Table 2. Comparison of BER with QPSK

\begin{tabular}{|l|c|c|l|}
\hline \multirow{2}{*}{ Schemes } & \multicolumn{2}{|c|}{ Eb/N0 } & \multirow{2}{*}{ PAPR(dB) } \\
\cline { 2 - 3 } & \multicolumn{2}{|c|}{ QPSK } & \\
\cline { 2 - 3 } & $\begin{array}{c}\text { BER }= \\
10-3\end{array}$ & $\begin{array}{c}\text { BER }= \\
10-4\end{array}$ & \\
\hline Original OFDM & 7.01 & 8.32 & 11.00 \\
\hline Nonlinear companding & 9.56 & 11.25 & 6.00 \\
\hline
\end{tabular}

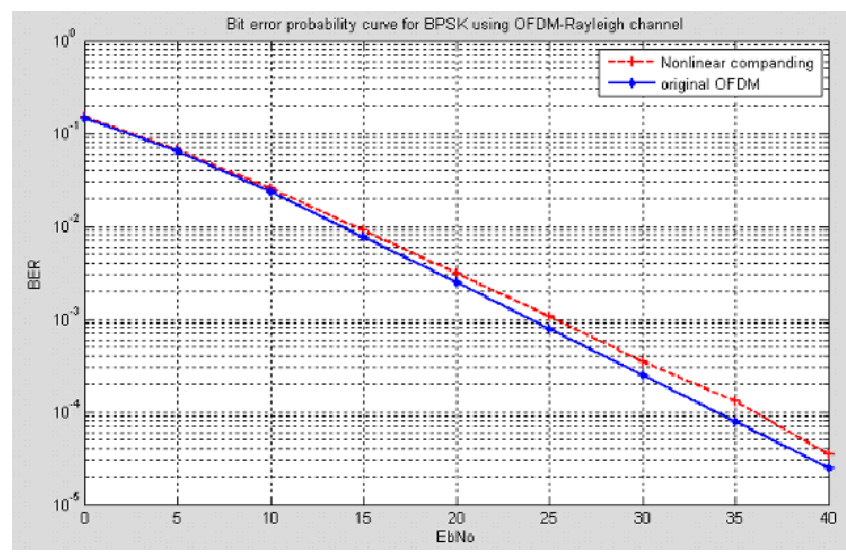

Figure 8. OFDM BER narration using BPSK.

vigorous sufficient in the fading canal (channel). The performance evaluation through the simulation shows that the proposed method process can suggests an excellent BER recital.

\section{Conclusion}

The designed system is used to develop the presentation of existing system proficiently with low deformation. The Simulation results shows that the proposed companding method possibly will provides an improved scheme recital in stipulations of PAPR lessening, and BER recital. 


\section{References}

1. Hou J, Jing L. Peak to average power ratio reduction of OFDM signals with nonlinear companding scheme. IEEE Transaction Broadcasting. 2010; 56(2):258-62 crossref
2. Zou WT, Wu Y. COFDM: An overview. IEEE Transaction Broadcasting. 1995 Mar; 41(1):1-8. crossref

3. Han SH, Lee JH. Modified selected mapping technique for PAPR reduction of coded OFDM signal. IEEE Transaction Broadcasting. 2004; 50(3):335-41. crossref 